\title{
CONTROLE DINÂMICO DE GAP ENTRE BARRAS NO LAMINADOR MORGAN DA VOTORANTIM SIDERURGIA*
}

\author{
Daniel Mesquita Duarte ${ }^{1}$ \\ Reginaldo Tenório da Silva² \\ Fabio Carvalho Mauricio ${ }^{3}$ \\ Alexandre Camargo Torres ${ }^{4}$ \\ Leandro Luís da Silva ${ }^{5}$
}

\section{Resumo}

A configuração física de equipamento do sistema de desenfornamento do laminador Morgan na unidade Resende, que é preparado para laminação em dois veios, torna o controle de GAP entre barras muito complexo. O sistema de controle original era suscetível a variações, acarretando na limitação do GAP mínimo na entrada do laminador em cinco segundos. Tendo como base as fontes de variação do projeto original, foi desenvolvido um novo sistema de controle que reduziu as variações em até $1700 \%$, reduzindo o GAP mínimo de cinco para dois segundos. No que tange à questão do GAP mínimo, o novo sistema de controle garante uma tolerância de 0,1 segundo. Este novo sistema pode ser dividido em duas partes: o controle e correção do GAP entre o forno e a primeira gaiola, e a correção de GAP no trem desbastador. Palavras-chave: Laminador; GAP entre barras; Sistema de controle; Automação.

\section{DYNAMIC GAP BETWEEN BARS CONTROL IN THE MORGAN ROLLING MILL OF VOTORANTIM SIDERURGIA}

\section{Abstract}

The equipment physical configuration of the furnace exit area in the hot rod rolling mill of Resende, which is prepared for a two strand rolling, makes the pacing control very complex. The original control system was susceptible to variation, resulting in limitation of the minimum GAP, at the rolling mill entrance, in five seconds. Based on the sources of variation of the original project, we developed a new control system that reduced the variations up to $1700 \%$, reducing the minimum GAP of five to two seconds. Regarding the issue of minimum GAP, the new control system ensures a tolerance of 0.1 second. This new system can be divided into two parts: the control and correction of the GAP between the furnace and the first rolling stand, and correction of GAP through the roughing mill.

Keywords: Rolling Mill; GAP between bars; Control system; Automation.

1 Consultor de Manutenção, Votorantim Siderurgia, Unidade Resende, Rio de Janeiro, RJ, Brasil.

2 Técnico Especialista em Processo, Votorantim Siderurgia, Unidade Resende, Rio de Janeiro, RJ, Brasil.

3 Operador IV, Votorantim Siderurgia, Unidade Resende, Rio de Janeiro, RJ, Brasil.

4 Coordenador de Manutenção e Oficina de Cilindros, Votorantim Siderurgia, Unidade Resende, Rio de Janeiro, RJ, Brasil.

5 Supervisor de Manutenção Elétrica, Votorantim Siderurgia, Unidade Resende, Rio de Janeiro, RJ, Brasil.

* Contribuição técnica ao $18^{\circ}$ Seminário de Automação e TI Industrial, 23 a 26 de setembro de 2014, São Paulo, SP, Brasil. 


\section{INTRODUÇÃO}

O GAP entre barras é o intervalo de tempo entre o fim da laminação de uma barra e o início da próxima, é um tempo em que não há produção no laminador, então deve ser reduzido ao máximo. Este trabalho surgiu da necessidade do aumento da produtividade do laminador Morgan na unidade Resende. Após a conclusão deste projeto também houveram ganhos na utilização e rendimento metálico, pois não ocorrem mais paradas no processo produtivo devido sucatas causadas por GAP entre barras muito curto, e também não há sucateamento parcial de barras para aumentar o GAP, devido um GAP abaixo do permitido.

\section{MATERIAIS E MÉTODOS}

Os equipamentos que compõem o setor de desenfornamento são: saída do forno de reaquecimento, mesa de transferência, pinch roll e tesoura snap. O setor de desenfornamento termina na primeira gaiola de laminação, conforme pode ser visto na figura 1.

A sequência de desenfornamento começa com o sinal de solicitação de tarugo ao forno, o forno executa um ciclo depositando um tarugo na mesa de rolos existente dentro do forno, a porta do forno abre e o tarugo entra na mesa de transferência. Quando o tarugo estiver completamente na mesa de transferência, a mesa executa um movimento transversal para posicionamento do tarugo no veio correto de laminação. Existe um sensor de detecção de metal quente (HMD - Hot Metal Detector) após a mesa de transferência que indica que o tarugo começou a sair da mesa, após um tempo é dado comando para fechar o pinch roll e é reduzida a velocidade do caminho de rolos, sincronizando as velocidades dos rolos e pincho roll com a velocidade de entrada da gaiola 1. Após a barra entrar na gaiola 1 é dado comando para abrir o pinch roll.

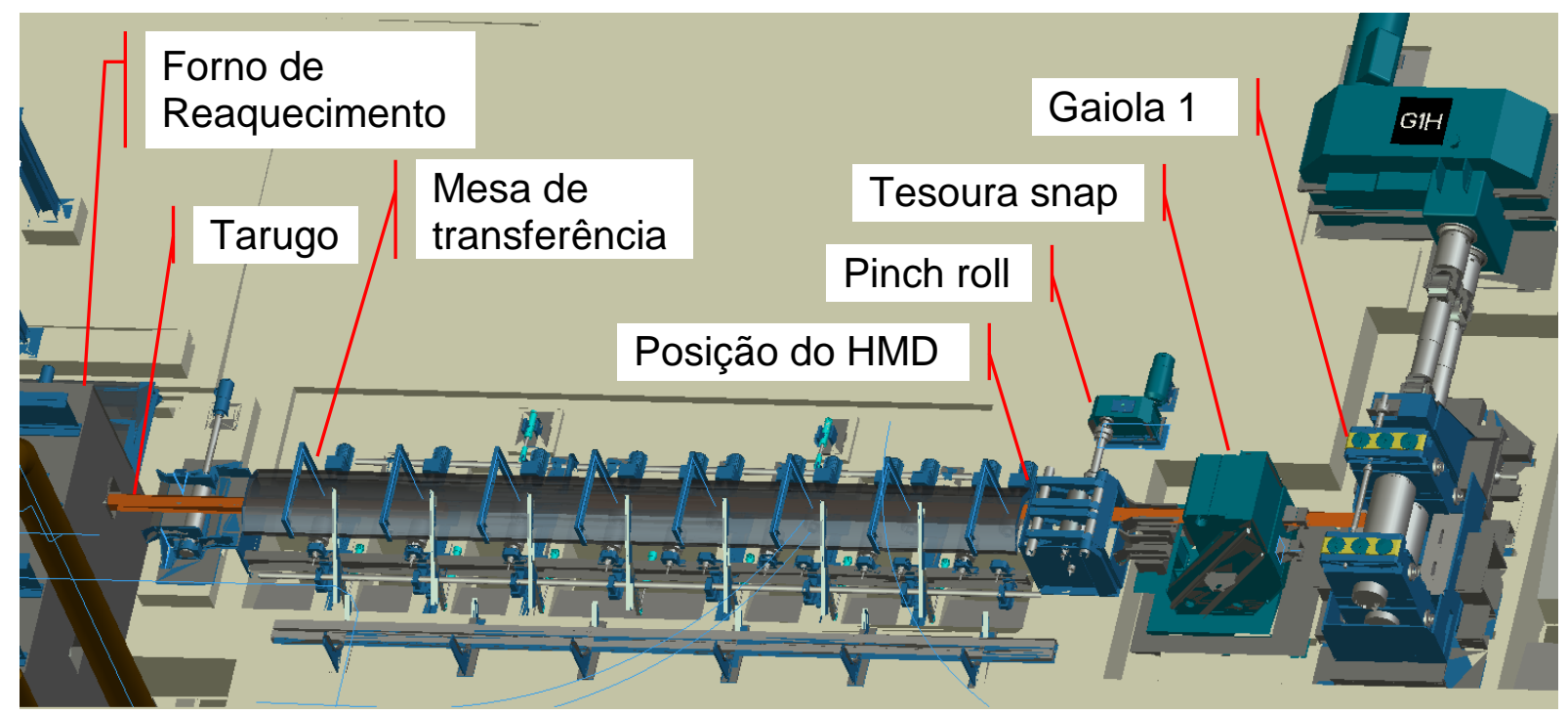

Figura 1. Equipamentos do setor do desenfornamento

* Contribuição técnica ao $18^{\circ}$ Seminário de Automação e TI Industrial, 23 a 26 de setembro de 2014, São Paulo, SP, Brasil. 


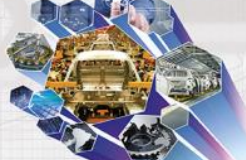

\subsection{Funcionamento do Sistema de Controle de GAP Antigo}

No laminador Morgan, o controle de GAP calcula o tempo que a barra demora para ser laminada completamente pela gaiola 1 (levando em consideração o comprimento do tarugo e a velocidade de entrada da gaiola 1) e também o tempo entre o pedido de tarugo para o forno e a chegada do mesmo na gaiola 1. Estes dois tempos são subtraídos e é somado o valor de GAP desejado, assim é obtido o tempo a ser esperado do momento que a cabeça da barra entra na gaiola 1 até o momento de enviar o comando para o sistema de controle do forno, solicitando um tarugo.

Foram observadas as seguintes variações que afetavam no GAP real:

1. Variação no comprimento do tarugo;

2. Variação do tempo de ciclo de desenfornamento do forno;

3. Variação do tempo que a barra demora a ir do forno até a gaiola 1 (variação de atrito nos rolos e agarramentos nas guias laterais);

4. Variação da velocidade da gaiola 1 pela cascata de velocidade (durante a barra toda).

\subsection{Funcionamento do Novo Controle de GAP}

A solicitação de tarugo é feita pelo controle de GAP antigo antecipada em 8 segundos (como se o GAP inserido no supervisório fosse 8 segundos menor). A posição da barra dentro da mesa de transferência é monitorada por integração de velocidade, e assim, 1,5 segundo antes da barra sair da mesa de transferência, a velocidade da mesa de rolos é reduzida de $1 \mathrm{~m} / \mathrm{s}$ para $0,25 \mathrm{~m} / \mathrm{s}$. Quando o tarugo atua o HMD após a mesa de transferência, a mesa de rolos é desligada, então conta-se o tempo do novo controle de GAP, mostrado na equação (4). O tempo do novo controle de GAP é o tempo que falta para a barra terminar de passar pela gaiola 1, equação (6), mais o valor de GAP desejado, mais uma correção dinâmica da variação do GAP, equação (5), menos o tempo que a barra irá demorar para ir da saída da mesa de transferência até a gaiola 1, equação (8). Após este tempo, a mesa de rolos acelera para $0,9 \mathrm{~m} / \mathrm{s}$ e depois reduz para a velocidade de entrada da gaiola 1 de tal forma que a barra esteja na velocidade de entrada da gaiola 1 aproximadamente $250 \mathrm{~mm}$ antes da gaiola 1 (ver figura 2). O tempo que a mesa de rolos deve ficar em $0,9 \mathrm{~m} / \mathrm{s}$ é calculado pela equação (9).

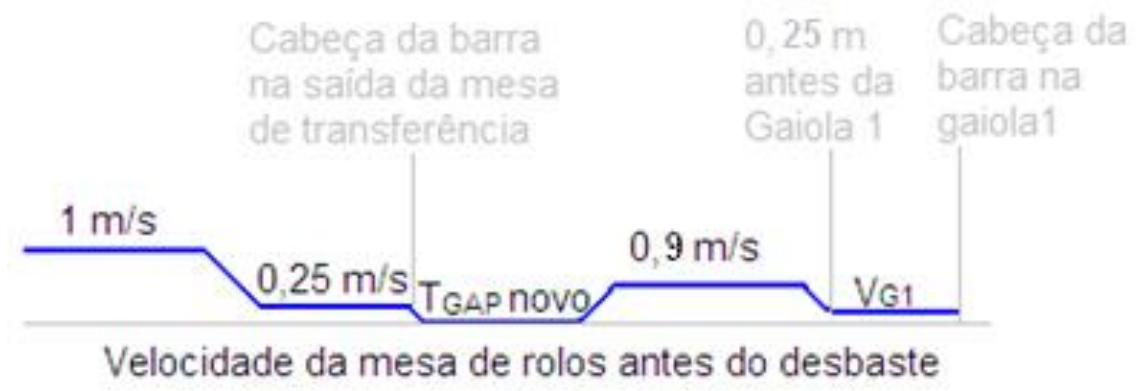

Figura 2. Nova configuração da sequência de valores de velocidade do caminho de rolos antes da gaiola 1

O detalhamento do cálculo do novo controle de GAP é descrito pelas equações abaixo:

$$
\text { Aceleração }=\text { Veloc }_{\text {Máx }} \times \text { Slope }
$$

\footnotetext{
* Contribuição técnica ao $18^{\circ}$ Seminário de Automação e TI Industrial, 23 a 26 de setembro de 2014 , São Paulo, SP, Brasil.
} 


$$
\begin{aligned}
& \text { Distância aceleração }=\frac{\text { Veloc }_{\text {Inicial }}+\text { Veloc }_{\text {Final }}}{2} \times \text { Tempo aceleração } \\
& \mathrm{T}_{\mathrm{GAP}}=\mathrm{T}_{\text {caudaR }}-\mathrm{T}_{\text {cabeça }}+\mathrm{GAP}+\mathrm{T}_{\text {corr }} \\
& \mathbf{T}_{\text {corr }}=\frac{(\text { OpInputGAP-ActGAP }) \times \text { FacGAPCor }}{2}
\end{aligned}
$$

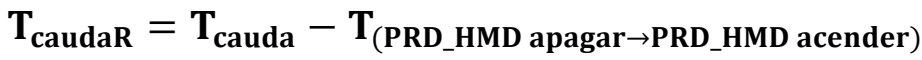

$$
\begin{aligned}
& \mathbf{T}_{\text {cauda }}=\frac{\text { StandMatDetDist }}{\text { EntrySpeed }} \\
& \mathbf{T}_{\text {cabeça }}=\mathbf{T}_{\text {aceleração }}+\mathbf{T}_{\mathbf{V}_{\text {alta }}}+\mathbf{T}_{\text {desaceleração }}+\mathbf{T}_{\mathbf{V}_{\text {baixa }}}
\end{aligned}
$$

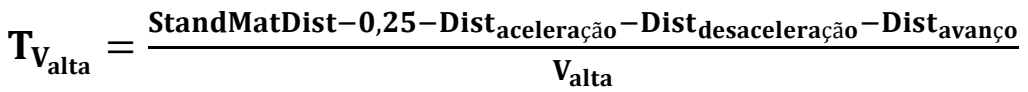

$$
\begin{aligned}
& \text { Dist }_{\text {aceleração }}=\frac{\mathrm{V}_{\text {alta }}}{2} \times \mathrm{T}_{\text {aceleração }} \\
& \text { Dist }_{\text {avanço }}=\frac{V_{\text {baixa1 }}}{2} \times \frac{V_{\text {baixa1 }}}{\text { Maxpeed } \times \text { Slope }} \\
& \mathbf{T}_{\text {desaceleração }}=\frac{\mid \mathrm{V}_{\text {alta }}-\text { EntrySpeed } \mid}{\text { MaxSpeed } \times \text { Slope }} \\
& \mathbf{T}_{\mathrm{V}_{\text {baixa }}}=\frac{0,25[\mathrm{~m}]}{\text { EntrySpeed }}
\end{aligned}
$$

Onde:

TcaudaR: Tempo para a cauda da barra terminar de passar pela gaiola 1;

Tcorr: Tempo de correção do GAP;

OplnputGAP: Set point do GAP inserido no supervisório;

ActGAP: Tempo de GAP entre barras lido na gaiola 1;

FracGAPCor: Fração para correção do GAP inserido no supervisório (valor típico: 10\%);

StandMatDist: Distância do HMD após a mesa de transferência até os cilindros da gaiola 1;

EntrySpeed: Velocidade de entrada da gaiola 1;

MaxSpeed x Slope: Resulta no valor de aceleração das mesas de rolos antes do desbaste;

$V_{\text {alta: }}$ 0,9 m/s;

Tempodifveloc: Tempo que será aumentado no GAP devido a redução de velocidade na gaiola;

Distavanço: Distância que a barra percorrerá depois que a cabeça chegar ao HMD após a mesa de transferência e for dado comando para parar a mesa de rolos

$V_{\text {baixa1: }}$ Velocidade quando a cabeça da barra está saindo da mesa de transferência $(0,25 \mathrm{~m} / \mathrm{s})$.

* Contribuição técnica ao $18^{\circ}$ Seminário de Automação e TI Industrial, 23 a 26 de setembro de 2014, São Paulo, SP, Brasil. 
No momento em que a barra chega ao HMD após a mesa de transferência, o caminho de rolos é desligado, e o tarugo passa alguns centímetros da posição do HMD devido à desaceleração do caminho de rolos, esta distância é calculada através da equação (11) e é utilizada no cálculo do tempo de GAP. Na figura 3 é mostrado um gráfico gerado através do software ibaPDA, onde é possível ver a referência de velocidade da mesa de rolos em azul, e a velocidade real do caminho de rolos em vermelho. A área do triângulo formado pelas linhas azul e vermelha é a distância gerada pela desaceleração mencionada acima.

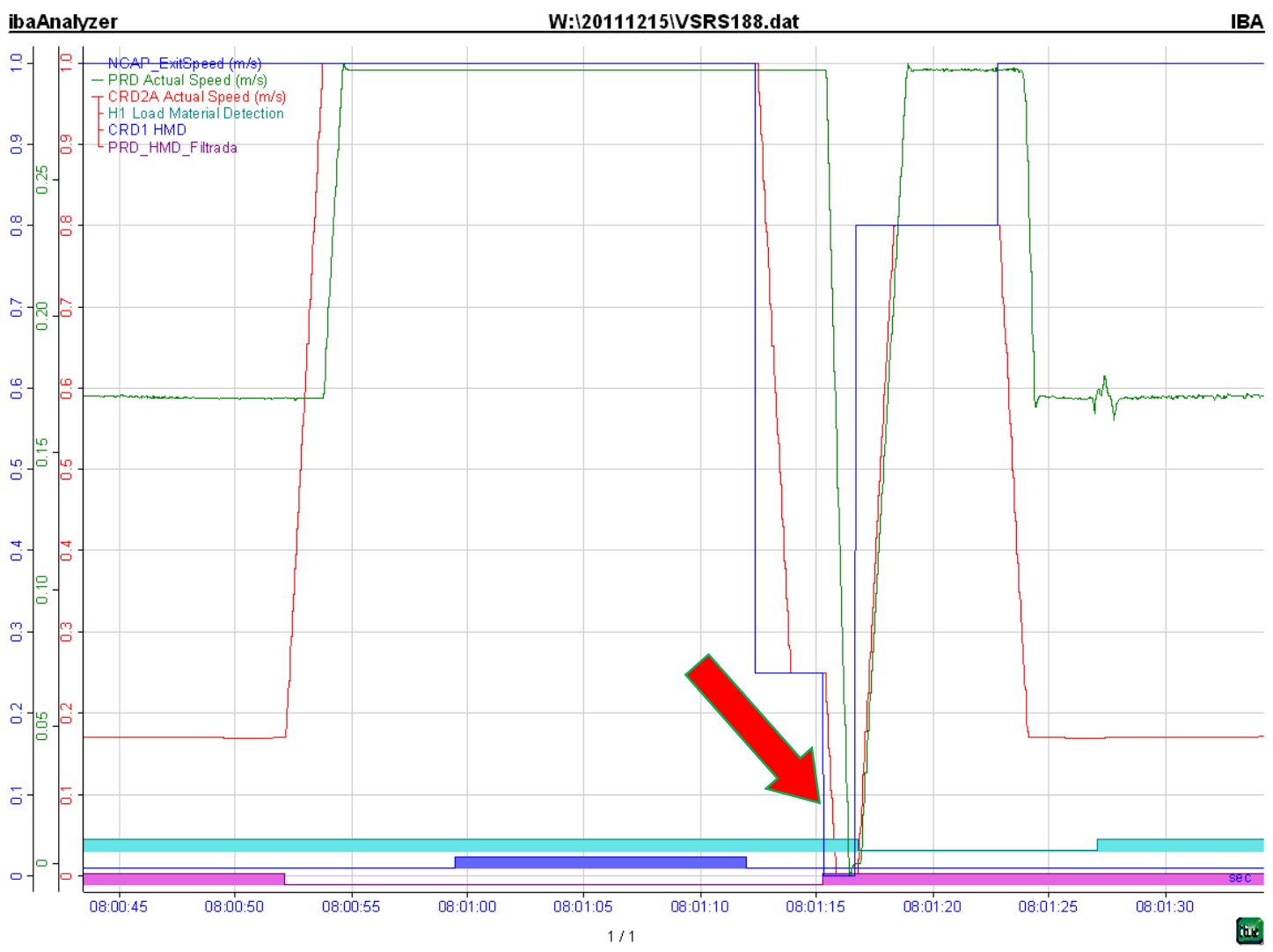

Figura 3. Análise da velocidade do caminho de rolos antes do desbaste

Variações que afetam no novo controle de GAP:

1. Variação do tempo que a barra demora a ir do forno até a gaiola 1 (variação de atrito nos rolos e agarramentos nas guias laterais);

2. Variação da velocidade da gaiola 1 pela cascata de velocidade (apenas na cauda).

\subsection{Funcionamento da Correção de GAP Curto no Desbaste}

Na correção de GAP curto no desbaste, quando a cabeça da barra entra na gaiola 1 é medido o GAP entre barras, se o GAP for menor do que o set point, é reduzida a velocidade da gaiola $1 \mathrm{em}$ até $10 \%$ da velocidade máxima do motor, por um tempo calculado em função da diferença entre GAP real e set point (ver figura 4). Primeiramente é calculada a distância necessária para o aumento do GAP (equação 19), considerando uma redução de velocidade de $10 \%$, se esta distância necessária for maior do que a distância disponível, então a disponível será utilizada ao invés da distância necessária para o cálculo do tempo que a gaiola deve ficar com velocidade

* Contribuição técnica ao $18^{\circ}$ Seminário de Automação e TI Industrial, 23 a 26 de setembro de 2014, São Paulo, SP, Brasil. 
reduzida (equação 20). Em todos os cálculos é levado em consideração o tempo e a distância necessários para desacelerar e acelerar o motor da gaiola.

A verificação e ajuste do GAP entre barras são feitas para as três primeiras gaiolas do laminador. A velocidade da gaiola em questão sempre está na velocidade normal de laminação antes de iniciar o controle automático de tração (alguns segundos antes da cabeça chegar à gaiola posterior).
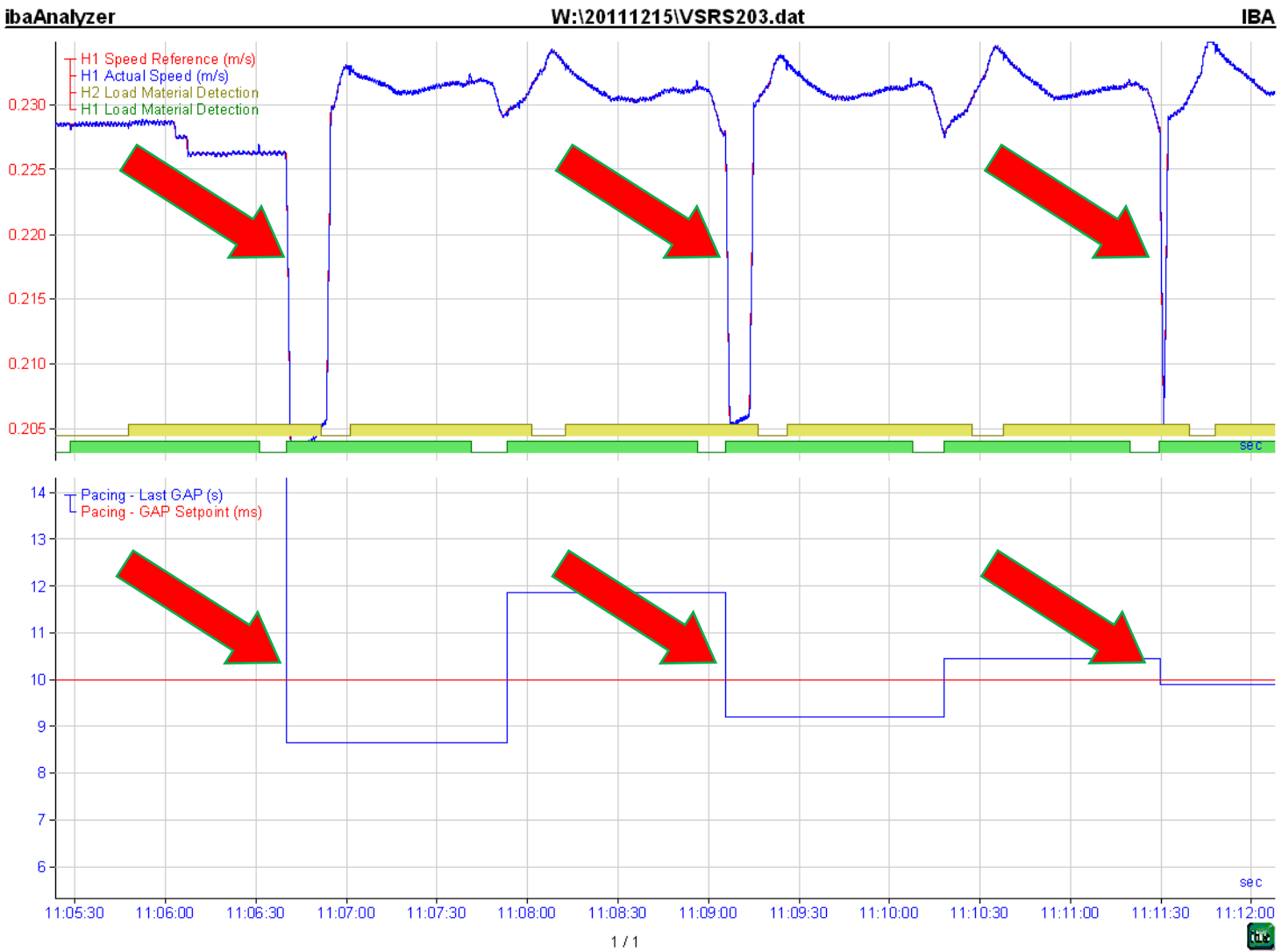

Figura 4. Funcionamento da correção de GAP curto no desbaste (no primeiro gráfico é mostrada a velocidade da gaiola 1 do desbaste e no segundo o GAP entre barras lido em azul e o set point em vermelho).

As equações utilizadas no sistema de correção de GAP curto no desbaste são descritas abaixo:

$$
\text { Tempo }_{\text {difVeloc }}=\mathbf{G A P}_{\text {setpoint }}-\mathrm{GAP}_{\text {lido }}+\text { IncGAP }_{\text {tração }}
$$

$$
\text { Tempo }_{\text {difVeloc }}=\text { Tempo }_{\text {Vreduzida }}+\text { Tempo }_{\mathrm{ac}}+\mathrm{Tempo}_{\text {desac }}-\text { Tempo }_{\text {Vnormal }}
$$

$$
\mathrm{Tempo}_{\text {desac }}=\frac{\left|\mathrm{V}_{\text {reduzida }}-\mathrm{Veloc}_{\text {normal }}\right|}{\text { Aceleração }}
$$

$$
\mathrm{Tempo}_{\mathrm{desac}}=\mathrm{Tempo}_{\mathrm{ac}}
$$

$$
\begin{aligned}
& \text { Tempo }_{\text {difVeloc }}=\frac{\text { Dist }}{V_{\text {reduzida }}}-\frac{\text { Dist }}{V_{\text {normal }}}+2 \times \text { Tempo }_{\text {desac }} \\
& \text { Dist }_{\text {necessária }}=\frac{\text { Tempo }_{\text {difVeloc }-2 \times \text { Tempo }_{\text {desac }}}}{\frac{1}{V_{\text {reduzida }}-\frac{1}{V_{\text {normal }}}}}
\end{aligned}
$$

* Contribuição técnica ao $18^{\circ}$ Seminário de Automação e TI Industrial, 23 a 26 de setembro de 2014, São Paulo, SP, Brasil. 


$$
\begin{aligned}
\text { Tempo }_{\text {Vreduzida }} & =\frac{\text { Dist }}{\mathrm{V}_{\text {reduzida }}} \\
\mathrm{V}_{\mathrm{G} 1} & =\frac{\mathrm{V}_{\mathrm{G} 2}}{\text { Fator } \mathrm{R}}
\end{aligned}
$$

Onde:

IncGAP tração: Valor medido do aumento do GAP entre barras no desbaste devido à tração;

Tempodifveloc: Tempo que vai ser aumentado no GAP devido a redução de velocidade na gaiola;

Tempovreduzida: Tempo que será necessário a velocidade da gaiola ficar reduzida para aumentar o GAP entre barras;

Distnecessária: Distância necessária para aumentar o GAP, considerando uma redução de velocidade de $10 \%$;

$\mathrm{V}_{\mathrm{G} 1}=\mathrm{V}_{\text {normal }}$ : Velocidade de saída da gaiola 1 .

Devido ao fato do trem desbastador trabalhar com certa tração entre as gaiolas, o GAP entre barras medido na gaiola 6 é maior do que o GAP medido na gaiola 1 . Este aumento de GAP devido tração é constantemente medido em cada gaiola e é efetuada uma média das 5 últimas medições para cada gaiola. Está média é considerada na lógica de correção do GAP nas gaiolas do desbaste.

\subsection{Correção de GAP Longo no Desbaste}

Como foram obtidos resultados muito bons na correção de GAP curto no desbaste (variação máxima de 0,1 segundo), foi desenvolvido um sistema similar, mas para correção de GAP longo. A diferença é que a gaiola é acelerada para reduzir o GAP, ao invés de reduzida a sua velocidade como é feita na correção de GAP curto.

A correção de GAP longo é ativada sempre que o GAP entre barras medido em uma gaiola do desbaste, somado ao aumento de GAP devido à tração entre as gaiolas, for maior do que o set point mais 0,5 segundo. Ou seja, o sistema irá corrigir sempre que o GAP entre barras for maior do que o set point, na saída do trem desbastador, em 0,5 segundo.

\section{RESULTADOS E DISCUSSÃO}

Nos gráficos das próximas figuras são feitas comparações da dispersão dos valores de GAP entre barras após o trem desbastador. Foram utilizadas quarenta amostras consecutivas de valores de GAP entre barras. Serão mostrados três gráficos das bitolas mais representativas no laminador. O gráfico com a indicação "Antigo" no título se refere às amostras com o sistema original de GAP, "Novo 1" indica novo sistema de controle de GAP e correção de GAP curto no desbaste e "Novo 2" são os mesmos sistemas do "Novo 1" com a adição da correção de GAP longo no desbaste.

Nos três gráficos da Figura 5 estava sendo produzida a bitola $6,50 \mathrm{~mm}$ fio máquina. No gráfico com título "Antigo" o set point de GAP era 5 segundos enquanto que nos gráficos "Novo 1" e "Novo 2" o set point estava em 4 segundos. Os gráficos estão na mesma escala

\footnotetext{
* Contribuição técnica ao $18^{\circ}$ Seminário de Automação e TI Industrial, 23 a 26 de setembro de 2014 ,
} São Paulo, SP, Brasil. 

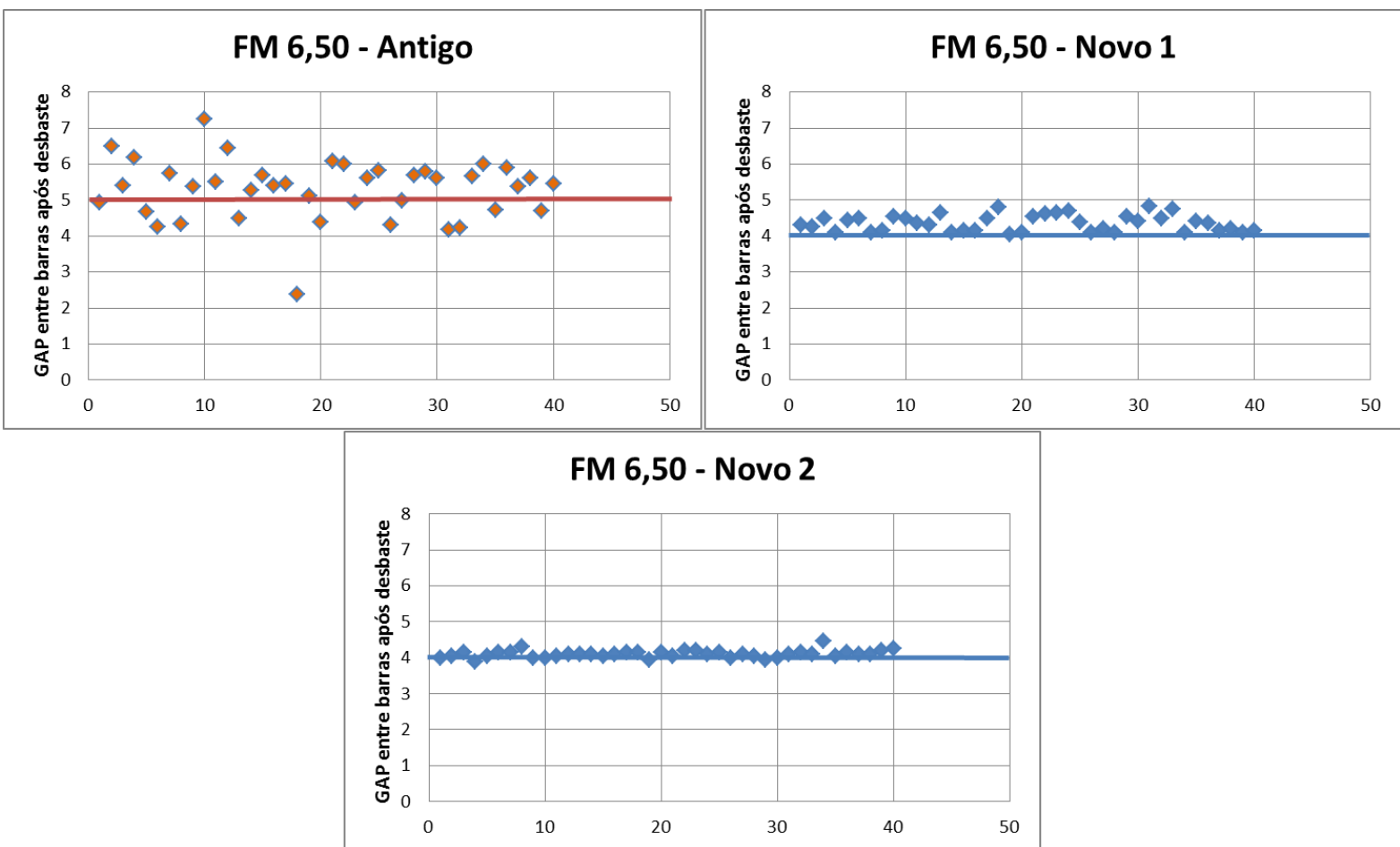

Figura 5. Comparação da dispersão do GAP na bitola de 6,50 mm fio máquina

Nos gráficos da figura 6 estava sendo produzida a bitola 5,50 $\mathrm{mm}$ fio máquina. Nos gráficos com título "Antigo" e "Novo 1" o set point de GAP era 5 segundos enquanto que no gráfico "Novo 2" o set point estava em 4 segundos.
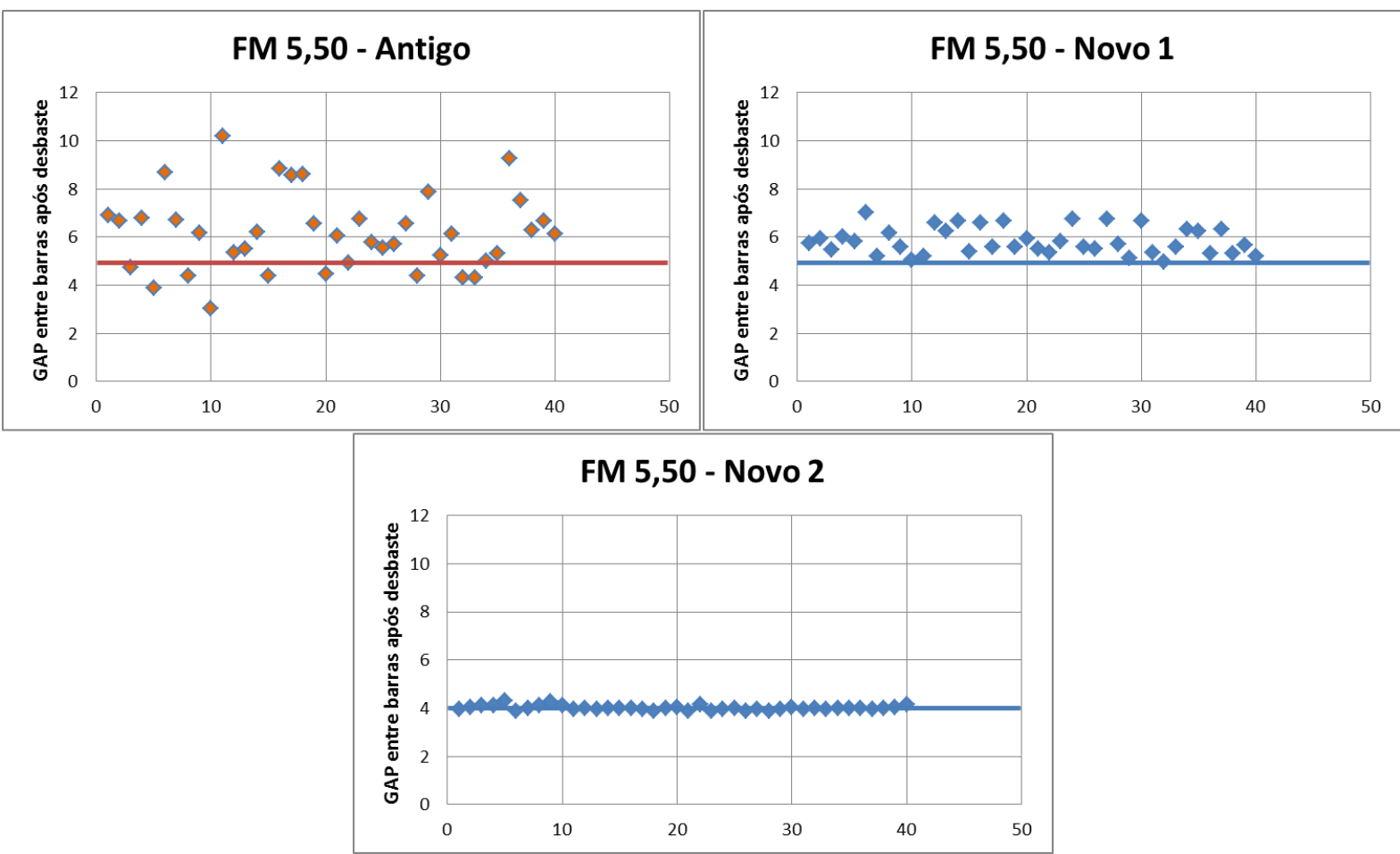

Figura 6. Comparação da dispersão do GAP na bitola de $5,50 \mathrm{~mm}$ fio máquina

Nos gráficos da Figura 7 estava sendo produzida a bitola $8,00 \mathrm{~mm}$ CA50. Nos gráficos com título "Antigo" e "Novo 1" o set point de GAP era 5 segundos enquanto que no gráfico "Novo 2" o set point estava em 4,6 segundos.

* Contribuição técnica ao $18^{\circ}$ Seminário de Automação e TI Industrial, 23 a 26 de setembro de 2014, São Paulo, SP, Brasil. 

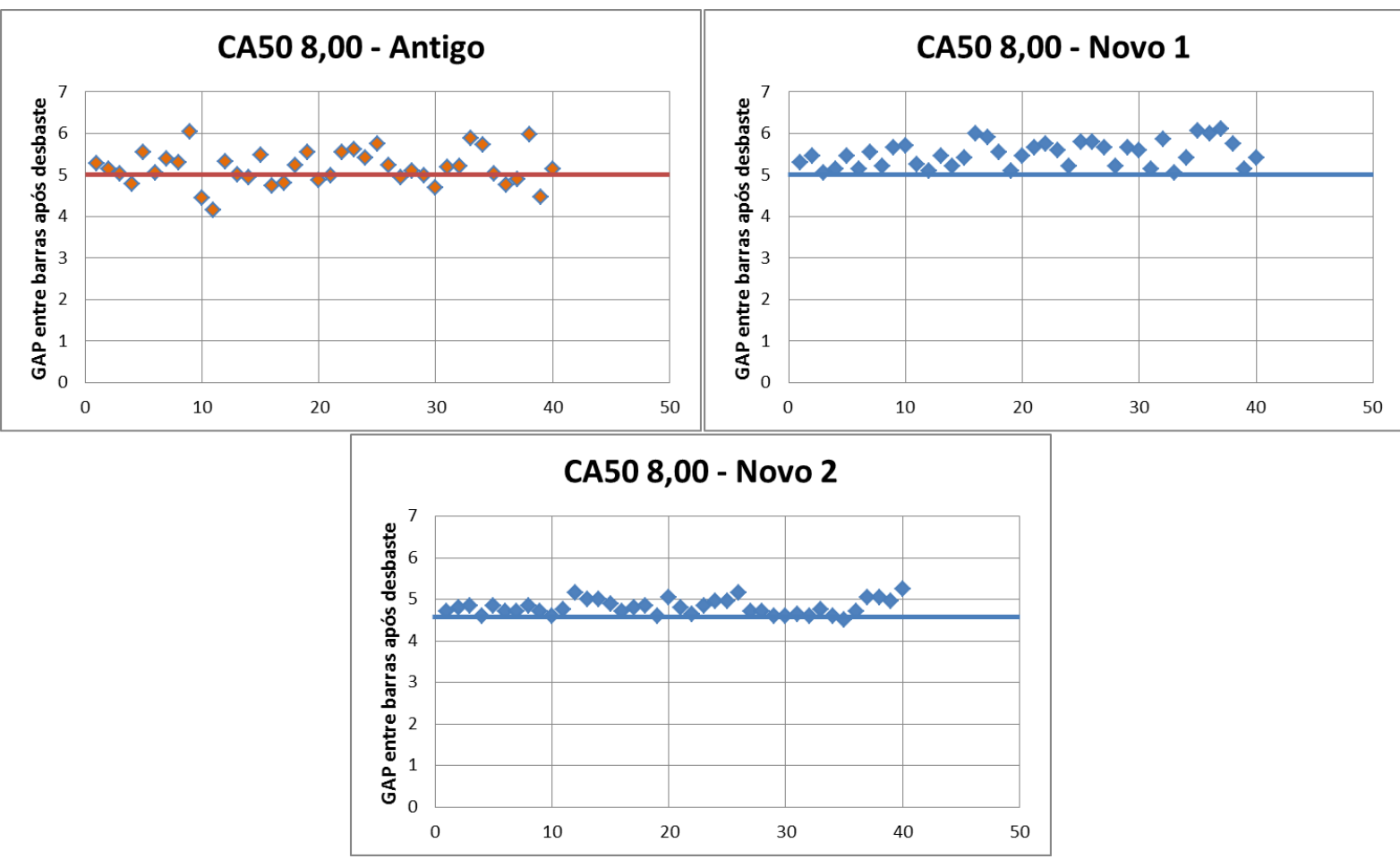

Figura 7. Comparação da dispersão do GAP na bitola de $8,00 \mathrm{~mm}$ nervurado

Nos gráficos da Figura 8 estava sendo produzida a bitola 10,00 mm CA50. O set point de GAP era 5 segundos em todos os gráficos
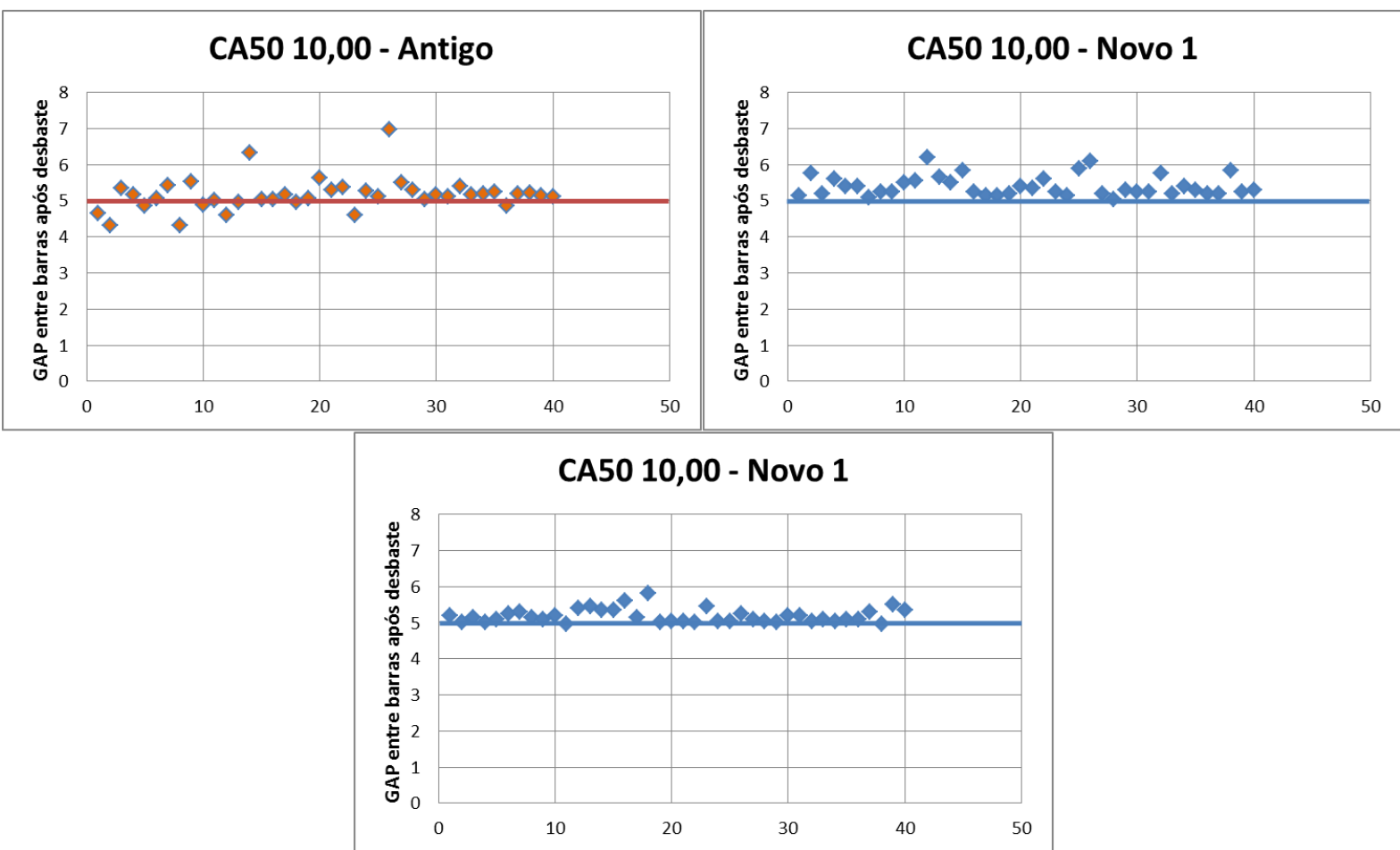

Figura 8. Comparação da dispersão do GAP na bitola de 10,00 mm nervurado

Foi possível observar que os sistemas de correção de GAP entre barras curto e longo no desbaste possui um desempenho melhor nos materiais que demandam uma velocidade mais baixa nas gaiolas do desbaste. Esta conclusão é coerente, pois com velocidade menor o tempo disponível para correção é maior.

* Contribuição técnica ao $18^{\circ}$ Seminário de Automação e TI Industrial, 23 a 26 de setembro de 2014, São Paulo, SP, Brasil. 
Na tabela 1 é possível verificar que houve uma redução na variação do GAP entre barras, principalmente nas bitolas de baixa velocidade no desbaste, sendo que houve melhora de até $1700 \%$.

Tabela 1. Comparação do desempenho do controle automático de GAP

\begin{tabular}{|c|c|c|c|c|c|c|c|c|c|c|c|c|}
\hline \multirow{2}{*}{ GAP } & \multicolumn{3}{|c|}{6,50} & \multicolumn{3}{|c|}{5,50} & \multicolumn{3}{|c|}{ 8,0 CA50 } & \multicolumn{3}{|c|}{10,0 CA50 } \\
\hline & Antigo & Novo 1 & Novo 2 & Antigo & Novo 1 & Novo 2 & Antigo & Novo 1 & Novo 2 & Antigo & Novo 1 & Novo 2 \\
\hline Set pont & 5 & 4 & 4 & 5 & 5 & 4 & 5 & 5 & 4,6 & 5 & 5 & 5 \\
\hline Mínimo & 2,39 & 4,04 & 3,9 & 3,03 & 4,95 & 3,9 & 4,16 & 5,04 & 4,5 & 4,31 & 5,05 & 4,95 \\
\hline Máximo & 7,25 & 4,84 & 4,45 & 10,2 & 7 & 4,3 & 6,04 & 6,1 & 5,25 & 6,96 & 6,2 & 5,8 \\
\hline Média & 5,28 & 4,36 & 4,10 & 6,16 & 5,83 & 4,01 & 5,16 & 5,50 & 4,81 & 5,17 & 5,41 & 5,19 \\
\hline Desv. Padrão & 0,841 & 0,231 & 0,100 & 1,606 & 0,560 & 0,092 & 0,416 & 0,305 & 0,183 & 0,457 & 0,282 & 0,190 \\
\hline
\end{tabular}

\section{CONCLUSÃO}

Com o desenvolvimento deste trabalho percebemos o potencial de ganho que melhorias em automação e controle são capazes de proporcionar, sem gastos com novos equipamentos ou serviços. Através de uma solução inovadora, conseguimos alcançar a meta estipulada: o GAP entre barras na tesoura 6, ou seja, após o trem desbastador, não ser menor do que o set point de GAP com tolerância de 0,1 segundos. Com este resultado conseguimos acabar com um limitante da produtividade do laminador, abrindo a possibilidade de galgarmos valores de GAP entre barras cada vez menores.

\section{Agradecimentos}

Gostaríamos de agradecer toda a equipe de operação e manutenção da Gerência de Laminação a Quente da unidade Resende da Votorantim Siderurgia, pelo auxílio técnico e na execução de testes.

\section{BIBLIOGRAFIA}

1 Máximo A, Alvarenga B. Curso de Física. Editora Harbra. 3ª edição. 1992. p 71-91; 123-145

2 IBA AG ibaPDA-V6 Manual. Versão 1.2 en. 2009

3 Siemens AG Statement List (STL) for S7-300 and S7-400 Programming Reference Manual. Versão 03/2003. 2003.

* Contribuição técnica ao $18^{\circ}$ Seminário de Automação e TI Industrial, 23 a 26 de setembro de 2014, São Paulo, SP, Brasil. 